\title{
Lattice Boltzmann simulation and fractal analysis of effective thermal conductivity in porous media
}

\author{
Xuan Qin ${ }^{\mathrm{a}, \mathrm{b}}$, Jianchao Cai ${ }^{\mathrm{a}^{*}}$, Yingfang Zhou ${ }^{\mathrm{b}^{*}}$, Zhiqin Kang ${ }^{\mathrm{b}, \mathrm{c}}$
}

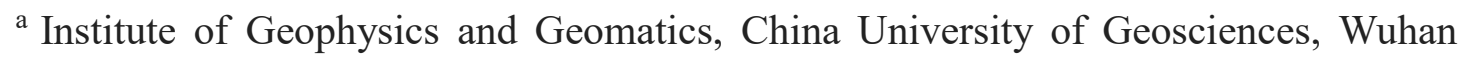
430074, P. R. China

b School of Engineering, University of Aberdeen, Aberdeen, AB243UE, United Kingdom

${ }^{c}$ Key Laboratory of In-situ Property-improving Mining of Ministry of Education, Taiyuan University of Technology, Taiyuan 030024, P. R. China

* Corresponding author: caijc@,cug.edu.cn (J. Cai); yingfang.zhou@abdn.ac.uk (Y. Zhou)

\section{Abstract}

Heat transfer in porous media has been attracting wide attention in a variety of scientific researches and engineering applications over the past decades. Due to the complexity of pore-solid microstructural features, an accurate simulation of heat transport process and evaluation of effective thermal conductivity in saturated porous media remain a great challenge. In this work, the lattice Boltzmann method (LBM) simulation of fluidsolid coupling heat transfer and a fractal model are adopted to study the thermal transport property in three-dimensional porous media. The accuracy and reliability of the proposed LBM simulation is validated by the effective thermal conductivity of a straight capillary tube, which analytical solution is available. The relation between effective thermal conductivity in porous media with different geometrical parameters based on the LBM simulation and fractal model are analyzed and discussed extensively. The result shows that the proposed LBM fluid-solid coupling simulation is an efficient tool to describe the heat transfer in complex porous media and the fractal dimension not only has an important effect on accurately estimating the effective thermal conductivity but also may be a useful parameter to determine the representative elemental volume (REV) of porous media. It is also found that compared with LBM simulation, the fractal model over predicates the effective thermal conductivity and the Maxwell model under predicates the effective thermal conductivity. The proposed LBM simulation and fractal model may reveal a better understanding for the thermophysical 
mechanisms of heat transfer in porous media.

Keywords: Effective thermal conductivity; Lattice Boltzmann method; Fractal model; Porous media 


\section{Introduction}

Fluid flow and heat transfer in porous media plays an essential role in a variety of scientific researches and engineering applications, such as geothermal energy extraction [1], petroleum engineering [2], nuclear waste subsurface disposal [3], hydrocarbon formation modeling [4], and civil engineering [5]. While due to the porous structure complexity, a rigorous theoretical description of transport behaviors remains a great challenge.

Since the fractal geometry theory was initially developed by Mandelbrot [6] to describe the complexity of the natural objects, it has been widely applied to model the transport process in porous media [7, 8]. Yu and Cheng [9] proposed an effective thermal conductivity model in bi-dispersed porous media based on fractal theory and thermoelectric analogy method. Miao et al. [10] presented an analytical solution of effective thermal conductivity in fractal dual-porosity media and found that the proposed model could provide a better understanding of the physical basis of heat transport in porous media than conventional models. Pia et al. [11] developed an intermingled fractal units model to study the relationship between the thermal properties and microstructure in ceramic porous media and the model predictions agree well with the experimental data. Jin et al. [12] proposed a fractal model to predict the effective thermal conductivity in porous media of the autoclaved aerated concrete based on the self-similar Sierpinski carpet. Recently, we have applied the fractal theory to describe the effect thermal conductivity in unsaturated porous media. The effectiveness and reliability of this proposed fractal model is validated by comparing with the published experimental data [13]. To the best of our knowledge, the parameters used in most of fractal models, such as the fractal dimension of pore size distribution, the fractal dimension of granular matrix size distribution, and the tortuosity fractal dimension, are normally estimated by empirical equations [14], and very limited effort has been covered to determine these parameters directly from available three-dimensional microCT rock images for most of the existing fractal based effective thermal conductivity models [15-17]. 
As the development of computing and advanced imaging techniques to capture the detailed porous structure, a large number of numerical methods have been proposed to study the fluid flow and heat transfer in porous media. Most of existing computational fluid dynamics (CFD) applied to simulate the transport behaviors in porous media include some conventional grid based numerical techniques, such as the finite difference method (FDM) [18], finite element method (FEM) [19], and finite volume method (FVM) [20]. Besides the grid based numerical methods, particle based approaches, for example the lattice Boltzmann method (LBM), have been proposed to simulate and model the fluid flow and heat transfer in porous media [21, 22]. The fundamental concept of the LBM is to utilize the particle groups, which are much bigger than the fluid molecules but much smaller than the real macroscopic scale, to describe the transport behaviors by calculating the interactions of the particle distribution function [23]. It is well evidenced that the LBM can not only recover the governing equations based on the Chapman-Enskog analysis but also account for the complicated boundaries of governing equations accurately [24]. Therefore, a significant number of researches on the transport properties in porous media have been successfully carried out based on the LBM. For example, Cai and Huai [25] simulated the fluid-solid conjugate heat transfer in fractal porous media based on the LBM. Basha et al. [26] applied the parallel LBM to model and simulate the fluid flow and heat transfer in rotating channels. Lamarti et al. [27] proposed a numerical study to simulate the mixed convection heat transfer in a cavity driven by periodically oscillating lid based on the LBM. Fang et al. [28] presented the LBM to predict the effective thermal conductivity for anisotropic heterogeneous porous media. They observed that the numerically predicted results are in good agreement with experiment data.

The accurate evaluation of the relation between heat transfer property and complex porous media is still a daunting task. Meanwhile, the parameters of fractal dimension used in most of previous fractal models are normally estimated by empirical equations. In this work, we present pore-scale LBM simulations of fluid-solid coupling heat transfer and fractal model to calculate the effective thermal conductivity in constructing 
three-dimensional cubes with different sizes and porosities from a micro-CT images of Berea sandstone core. The proposed LBM simulation is first validated by the benchmark of analytical solution results. The impacts of simulation size, porosity and rock fluid thermal conductivity ratio on the effect thermal conductivity of Berea sandstone are investigated and discussed extensively. The present LBM simulation and fractal model may reveal the better understanding of physical basis for heat transport in porous media.

\section{Materials and samples constructing}

In this work, the three-dimensional micro-CT images of Berea sandstone core are used to study the relation between effective thermal conductivity and different microstructures of rock porous media based on the LBM and available fractal model [13]. The Berea sandstone is a sedimentary rock and has been widely applied to investigate the transport properties of reservoir rock for the past three decades [29]. The petrophysical parameters of selected Berea sandstone core are given in the Table 1. After the micro-CT images processing, the binary images are obtained (see Fig. 1). Fig. 1 also presents the process of constructing the samples of three-dimensional cubes with different sizes and porosities. As shown in Fig. 1, the different colors of threedimensional cube represent the different sizes of constructing samples. The sizes of samples are $50^{3}, 100^{3}, 150^{3}, 200^{3}, 250^{3}$, and $300^{3}$ voxels, respectively. In addition, the 63 different three-dimensional cubes with the size of $100^{3}$ pixels can be generated by this selected Berea sandstone core $(300 \times 300 \times 750$ pixels $)$. The samples with different porosities can be generated by these 63 different cubes.

Table 1. The petrophysical parameters of selected Berea sandstone core.

\begin{tabular}{llll}
\hline Sample & Size (pixels) & Resolution $(\mu \mathrm{m})$ & Porosity \\
\hline Berea sandstone & $300 \times 300 \times 750$ & 4.9 & 0.1917 \\
\hline
\end{tabular}




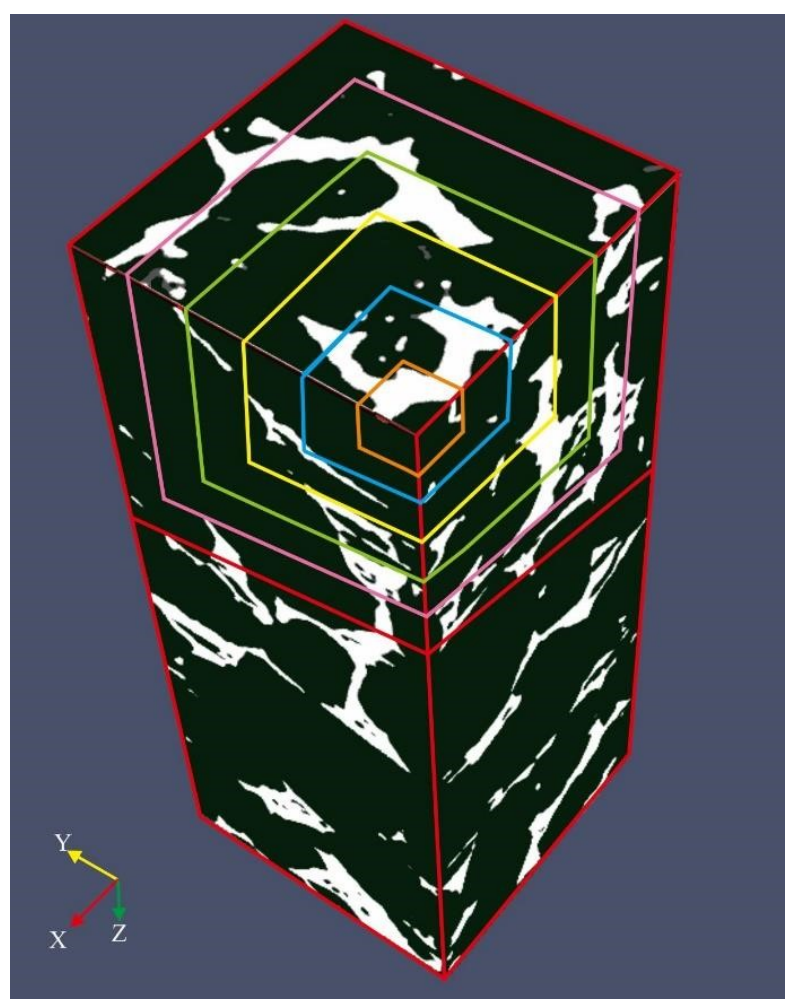

Fig. 1. The geometry of binary three-dimensional Berea sandstone image and the samples constructing with different sizes and porosities. The color of white and black are pore phase and solid phase, respectively.

\section{A fractal model of effective thermal conductivity}

Recently, we have derived a new fractal model of effective thermal conductivity in unsaturated porous media originated from the Laplace's equation and it can be simplified to for saturated porous media as following [13]

$$
k_{e}=k_{f}+\frac{\left(D_{s}-1\right)^{3} \phi}{D_{s}^{2}\left(3-D_{s}\right)} \frac{3 k_{f}\left(k_{s}-k_{f}\right)}{k_{s}+2 k_{f}}
$$

where $k_{e}$ is the effective thermal conductivity of porous media, $D_{s}$ is the fractal dimension of grains size distribution, $\phi$ is the porosity, $k_{s}$ is the thermal conductivity of solid phase, and $k_{f}$ is the thermal conductivity of pore phase. This new fractal model of effective thermal conductivity can provide an explanation of physical basis 
for heat transfer. Please refer our recent publication for more details [13]. However, in our previous work, $D_{s}$ is estimated by the empirical equation, and it lacks of direct measurement from three-dimensional micro-CT images of porous media.

The box counting method is the most effective and well accepted approach to calculate the fractal dimension from the rock images [30-33]. Here we have adopted the algorithm proposed by $\mathrm{Wu}$ et al. [30] to estimate fractal dimension from threedimensional rock images; this method has been used in three-dimensional coal microfractures and it contains three main procedures to calculate the fractal dimension of a three-dimensional rock image based on the box counting algorithm. Firstly, the boxes of different length scales $r$ are selected to divide the binary images. Secondly, the number $N(r)$ of total boxes covering the granular matrix is computed under the different scale $r$. Thirdly, the obtained datasets $[\log (1 / r), \log N(r)]$ are linear fitted and the slop is the fractal dimension. In order to validate the box counting program, the self-similar Menger Sponge is used to calculate fractal dimension. As shown in Fig. 2, the result of the calculated fractal dimension from our program is identical with the theoretical value of $\log 20 / \log 3 \approx 2.7268$. This clearly demonstrates the validity of proposed program to determine the fractal dimension from three-dimensional images.

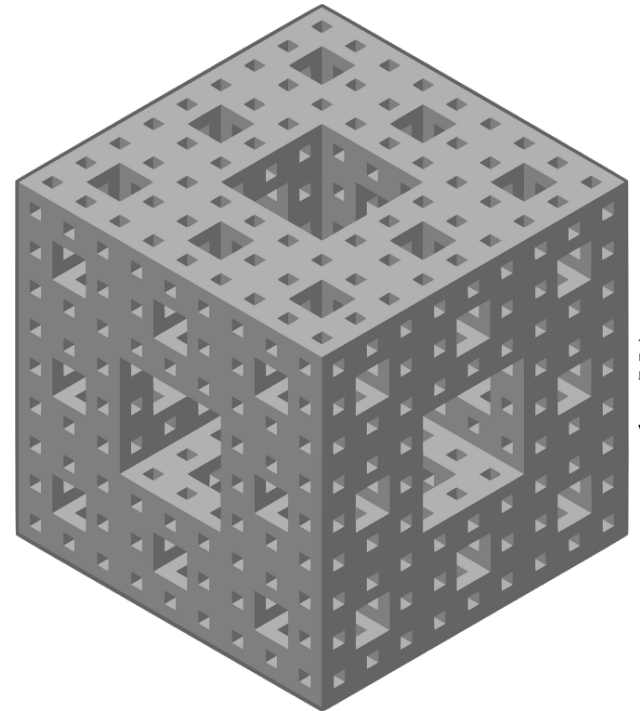

(a)

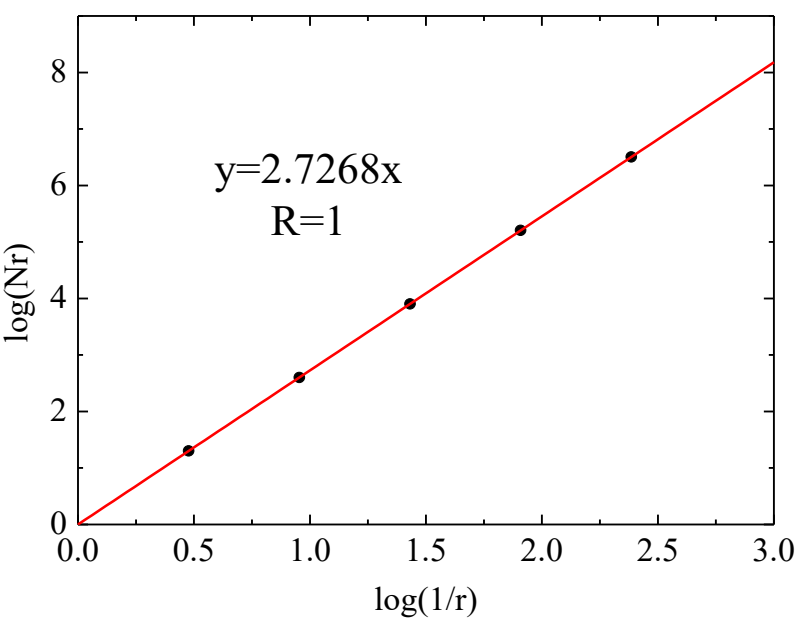

(b) 
Fig. 2. The fractal dimension of the Menger Sponge. (a) The schematic diagram of the Menger Sponge. (b) The result of calculated fractal dimension based on the box counting method.

\section{Numerical methodology}

\subsection{Lattice Boltzmann Method}

The three-dimensional nineteen-speed (D3Q19) LBM (see Fig. 3) with MultiRelaxation-Time (MRT) is used to model the steady state flow in porous media. The MRT scheme is presented not only to have a high accuracy result and better numerical stability [34] but also to eliminate spurious velocities near boundaries [35] compared with the Single-Relaxation-Time (SRT) LBM. To solve the heat transfer in porous media, the temperature field is simulated by the second distribution function as a passive scalar [36]. The double evolution equation with MRT collision model and a Bhatnagar-Gross-Krook (BGK) collision model for the velocity field and temperature field are written as in discretized form [35, 37]

$$
\begin{gathered}
f_{i}\left(\mathbf{x}+\mathbf{e}_{i} \delta t, t+\delta t\right)-f_{i}(\mathbf{x}, t)=-\mathbf{M}^{-1} \mathbf{S}\left[\left(m_{i}(\mathbf{x}, t)-m_{i}^{e q}(\mathbf{x}, t)\right)\right] \\
g_{i}\left(\mathbf{x}+\mathbf{e}_{i} \delta t, t+\delta t\right)-g_{i}(\mathbf{x}, t)=-\frac{1}{\tau_{g}}\left[g_{i}(\mathbf{x}, t)-g_{i}^{e q}(\mathbf{x}, t)\right]
\end{gathered}
$$

where $f_{i}$ is the particle density distribution function, $\mathbf{M}$ is the transformation matrix, $m_{i}=\mathbf{M} \cdot f_{i}$ is the velocity moments, $m_{i}^{e q}=\mathbf{M} \cdot f_{i}^{e q}$ is the equilibrium moments, $\mathbf{S}$ is the relaxation matrix, $g_{i}$ is the internal energy distribution function, $g_{i}^{e q}$ is the equilibrium internal energy distribution function, and $\tau_{g}$ is the internal energy relaxation parameter related to the thermal diffusivity. As shown in Fig. 3, the discrete velocity $\mathbf{e}_{i}$ for the D3Q19 LBM can be given by [38]

$$
\mathbf{e}_{i}= \begin{cases}(0,0,0), & i=0 \\ ( \pm 1,0,0) c,(0, \pm 1,0) c,(0,0, \pm 1) c, & i=1,2, \cdots 6 \\ (0, \pm 1, \pm 1) c,( \pm 1,0, \pm 1) c,( \pm 1, \pm 1,0) c, & i=7,8, \cdots 18\end{cases}
$$


where $c=\delta x / \delta t$ is the lattice speed.

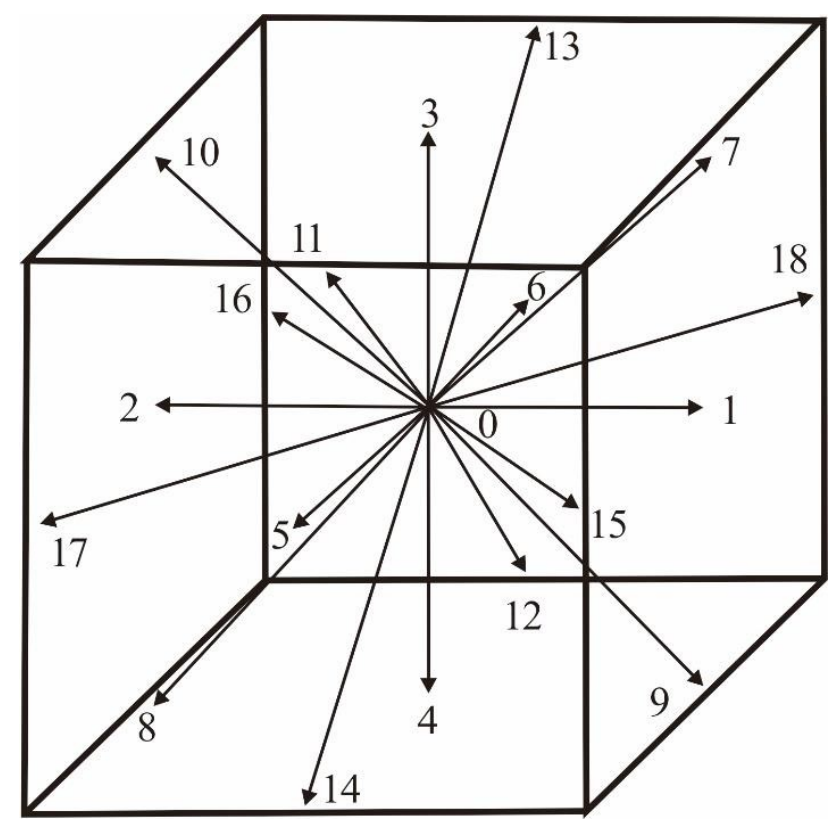

Fig. 3. The discretized lattice velocities of D3Q19.

The macroscopic parameters of density $\rho$ and velocity $\mathbf{u}$ are relevant to the particle density distribution function and can be written as [35]

$$
\rho=\sum_{i} f_{i}, \rho \mathbf{u}=\sum_{i} f_{i} \mathbf{e}_{i}
$$

The transformation matrix $\mathbf{M}$ can be written as [35, 39] 


$$
\mathbf{M}=\left[\begin{array}{ccccccccccccccccccc}
1 & 1 & 1 & 1 & 1 & 1 & 1 & 1 & 1 & 1 & 1 & 1 & 1 & 1 & 1 & 1 & 1 & 1 & 1 \\
-30 & -11 & -11 & -11 & -11 & -11 & -11 & 8 & 8 & 8 & 8 & 8 & 8 & 8 & 8 & 8 & 8 & 8 & 8 \\
12 & -4 & -4 & -4 & -4 & -4 & -4 & 1 & 1 & 1 & 1 & 1 & 1 & 1 & 1 & 1 & 1 & 1 & 1 \\
0 & 1 & -1 & 0 & 0 & 0 & 0 & 1 & -1 & 1 & -1 & 0 & 0 & 1 & -1 & 1 & -1 & 0 & 0 \\
0 & -4 & 4 & 0 & 0 & 0 & 0 & 1 & -1 & 1 & -1 & 0 & 0 & 1 & -1 & 1 & -1 & 0 & 0 \\
0 & 0 & 0 & 1 & -1 & 0 & 0 & 1 & -1 & 0 & 0 & 1 & -1 & -1 & 1 & 0 & 0 & 1 & -1 \\
0 & 0 & 0 & -4 & 4 & 0 & 0 & 1 & -1 & 0 & 0 & 1 & -1 & -1 & 1 & 0 & 0 & 1 & -1 \\
0 & 0 & 0 & 0 & 0 & -1 & -1 & 0 & 0 & -1 & -1 & 1 & -1 & 0 & 0 & -1 & 1 & -1 & 1 \\
0 & 0 & 0 & 0 & 0 & 4 & 4 & 0 & 0 & -1 & -1 & 1 & -1 & 0 & 0 & -1 & 1 & -1 & 1 \\
0 & 2 & 2 & -1 & -1 & -1 & -1 & 1 & 1 & 1 & 1 & -2 & -2 & 1 & 1 & 1 & 1 & -2 & -2 \\
0 & -4 & -4 & 2 & 2 & 2 & 2 & 1 & 1 & 1 & 1 & -2 & -2 & 1 & 1 & 1 & 1 & -2 & -2 \\
0 & 0 & 0 & 1 & 1 & -1 & -1 & 1 & 1 & -1 & -1 & 0 & 0 & 1 & 1 & -1 & -1 & 0 & 0 \\
0 & 0 & 0 & -2 & -2 & 2 & 2 & 1 & 1 & -1 & -1 & 0 & 0 & 1 & 1 & -1 & -1 & 0 & 0 \\
0 & 0 & 0 & 0 & 0 & 0 & 0 & 1 & 1 & 0 & 0 & 0 & 0 & -1 & -1 & 0 & 0 & 0 & 0 \\
0 & 0 & 0 & 0 & 0 & 0 & 0 & 0 & 0 & 0 & 0 & 1 & 1 & 0 & 0 & 0 & 0 & -1 & -1 \\
0 & 0 & 0 & 0 & 0 & 0 & 0 & 0 & 0 & 1 & 1 & 0 & 0 & 0 & 0 & -1 & -1 & 0 & 0 \\
0 & 0 & 0 & 0 & 0 & 0 & 0 & 1 & -1 & -1 & 1 & 0 & 0 & 1 & -1 & -1 & 1 & 0 & 0 \\
0 & 0 & 0 & 0 & 0 & 0 & 0 & -1 & 1 & 0 & 0 & 1 & -1 & 1 & -1 & 0 & 0 & 1 & -1 \\
0 & 0 & 0 & 0 & 0 & 0 & 0 & 0 & 0 & 1 & -1 & -1 & 1 & 0 & 0 & -1 & 1 & 1 & -1
\end{array}\right]
$$

The velocity moments are correspondingly given by $[35,37]$

$$
\mathbf{m}=\left(\rho, e_{k}, \varepsilon_{k}, j_{x}, q_{x}, j_{y}, q_{y}, j_{z}, q_{z}, 3 p_{x x}, 3 \pi_{x x}, p_{w w}, \pi_{w w}, p_{x y}, p_{y z}, p_{x z}, m_{x}, m_{y}, m_{z}\right)^{T}
$$

The equilibrium moments are determined by [35-37]

$$
\begin{aligned}
\mathbf{m}^{e q}= & \left(\rho,-11 \rho+19 \frac{j_{x}^{2}+j_{y}^{2}+j_{z}^{2}}{\rho_{0}}, 3 \rho-\frac{11}{2} \frac{j_{x}^{2}+j_{y}^{2}+j_{z}^{2}}{\rho_{0}}, j_{x},-\frac{2}{3} j_{x}, j_{y}, j_{z},-\frac{2}{3} j_{z},\right. \\
& \left.\frac{2 j_{x}^{2}-\left(j_{y}^{2}+j_{z}^{2}\right)}{\rho_{0}},-\frac{2 j_{x}^{2}-\left(j_{y}^{2}+j_{z}^{2}\right)}{2 \rho_{0}}, \frac{j_{y}^{2}-j_{z}^{2}}{\rho_{0}},-\frac{j_{y}^{2}-j_{z}^{2}}{2 \rho_{0}}, \frac{j_{x} j_{y}}{\rho_{0}}, \frac{j_{y} j_{z}}{\rho_{0}}, \frac{j_{x} j_{z}}{\rho_{0}}, 0,0,0\right)^{T}
\end{aligned}
$$

where $j_{x}=\rho u_{x}, j_{y}=\rho u_{y}, j_{z}=\rho u_{z}$, and the constant $\rho_{0}$ is the mean density in the fluid system [37].

The relaxation matrix $\mathbf{S}$ is a diagonal matrix and can be expressed as [35, 39]

$$
\mathbf{S}=\operatorname{diag}\left(0, s_{1}, s_{2}, 0, s_{4}, 0, s_{4}, 0, s_{4}, s_{9}, s_{10}, s_{9}, s_{10}, s_{13}, s_{13}, s_{13}, s_{16}, s_{16}, s_{16}\right)
$$

Then, the unknown parameters on the relaxation matrix can be determined by [ 35 , $37,40]$

$$
\left\{\begin{array}{l}
s_{1}=1.19 ; s_{2}=s_{10}=1.4 ; \\
s_{4}=1.2 ; s_{16}=1.98 ; \\
s_{9}=s_{13}=1 /(3 v+0.5)
\end{array}\right.
$$


where $v$ is the kinematic viscosity.

For the internal energy distribution function in the Eq. (3), the internal energy relaxation parameter $\tau_{g}$ is related to the thermal diffusivity $\alpha$ can be written as [36, 41]

$$
\alpha=\frac{5}{9}\left(\tau_{g}-\frac{1}{2}\right) \delta t
$$

where $\alpha=k / \rho c_{p} . k$ is the thermal conductivity of solid or fluid phase and $c_{p}$ is the specific heat capacity.

The equilibrium internal energy distribution function for the fluid phase can be expressed as $[36,41]$

$$
g_{i}^{e q}= \begin{cases}-\frac{\rho \varepsilon}{2} \frac{\mathbf{u} \cdot \mathbf{u}}{c^{2}}, & i=0 \\ \frac{\rho \varepsilon}{18}\left[1+\frac{\mathbf{e}_{i} \cdot \mathbf{u}}{c^{2}}+\frac{9\left(\mathbf{e}_{i} \cdot \mathbf{u}\right)^{2}}{2 c^{4}}-\frac{3 \mathbf{u} \cdot \mathbf{u}}{2 c^{2}}\right], & i=1,2, \cdots 6 \\ \frac{\rho \varepsilon}{36}\left[2+4 \frac{\mathbf{e}_{i} \cdot \mathbf{u}}{c^{2}}+\frac{9\left(\mathbf{e}_{i} \cdot \mathbf{u}\right)^{2}}{2 c^{4}}-\frac{3 \mathbf{u} \cdot \mathbf{u}}{2 c^{2}}\right], & i=7,8, \cdots 18\end{cases}
$$

As the velocity $\mathbf{u}$ in the solid phase is zero, Eq. (12) can be simplified by [21]

$$
g_{i}^{e q}= \begin{cases}0, & i=0 \\ \frac{\rho \varepsilon}{18}, & i=1,2, \cdots 18\end{cases}
$$

where the internal energy $\quad \varepsilon$ is related to the macroscopic temperature $T$ and can be defined as [41]

$$
\rho \varepsilon=\frac{3}{2} \rho R T=\sum_{i} g_{i}
$$

where $R$ is the gas constant.

4.2. Boundary conditions

4.2.1. Boundary treatment for fluid flow

The pressure boundary conditions are implemented to solve the inlet and outlet of 
fluid flow by means of the Zou-He model [42]. For the solid-fluid boundary, the half way bounce back model proposed by Ziegler [43] has been the most convenient and powerful strategy to deal with the no-slip fluid-solid interfaces, which has the secondorder accuracy. The working principle is that the distribution functions reaching the solid wall during propagation are reflected back into the original fluid field along the reversed incoming direction. The unknown distribution function at the solid boundary $f_{\bar{i}}$ can be determined by [43]

$$
f_{\bar{i}}\left(\mathbf{x}_{b}, t+\delta t\right)=f_{i}^{*}\left(\mathbf{x}_{b}, t\right)
$$

where $f_{i}^{*}$ is the post collision distribution function of moving toward the solid boundary. This standard bounce back model describe the solid boundary surface located at the middle of two neighboring lattice nodes with the same distance $\delta x / 2$. For the case when the boundary is not exactly placed at the $\delta x / 2$, the interpolated bounce back model was developed by Bouzidi et al. [44] to solve the arbitrary boundary shapes. The detailed cases of interpolated bounce back model are illustrated in Fig. 4. The quantity $h$ is defined to be the ratio of the boundary distance to a mesh with $0 \leq h<1$. The linear interpolation model for the unknown density populations can be given by [44]

$$
f_{\bar{i}}\left(\mathbf{x}_{b}, t+\delta t\right)= \begin{cases}2 h f_{i}^{*}\left(\mathbf{x}_{b}, t\right)+(1-2 h) f_{i}^{*}\left(\mathbf{x}_{b}-\mathbf{e}_{i}, t\right) & 0 \leq h<1 / 2 \\ \frac{1}{2 h} f_{i}^{*}\left(\mathbf{x}_{b}, t\right)+\frac{2 h-1}{h} f_{\bar{i}}^{*}\left(\mathbf{x}_{b}, t\right) & 1 / 2 \leq h<1\end{cases}
$$

\subsubsection{Boundary treatment for heat transfer}

For the samples of three-dimensional cube, the two surface boundaries are employed to be different constant temperature based on the Dirichlet boundary condition. But other boundaries are assumed to be adiabatic. The Dirichlet boundary condition for the unknown energy distribution function $g_{\bar{i}}$ can be expressed as [45]

$$
g_{\bar{i}}\left(\mathbf{x}_{b}, t+\delta t\right)=-g_{i}^{*}\left(\mathbf{x}_{b}, t\right)+\varepsilon_{B} \varphi_{B}
$$


where $g_{i}^{*}$ is the post collision energy distribution function of moving toward the boundary, $\varepsilon_{B}=1 / 9$ for D3Q19, and the energy source term $\varphi_{B}$ can be written as [36]

$$
\varphi_{B}=\frac{3}{2} \rho_{B} R T_{B}
$$

where $\rho_{B}$ is the density at the boundary and $T_{B}$ is the boundary temperature. Analogous to the solid-fluid boundary treatment of fluid flow as shown in Fig. 4, the thermal boundary based on the bounce back scheme and interpolation of the energy distribution function for the Dirichlet boundary condition is given by [46]

$$
g_{\bar{i}}\left(\mathbf{x}_{b}, t+\delta t\right)= \begin{cases}-2 h g_{i}^{*}\left(\mathbf{x}_{b}, t\right)+(2 h-1) f_{i}^{*}\left(\mathbf{x}_{b}-\mathbf{e}_{i}, t\right)+\varepsilon_{B} \varphi_{B} & 0 \leq h<1 / 2 ; \\ -\frac{1}{2 h} g_{i}^{*}\left(\mathbf{x}_{b}, t\right)+\frac{2 h-1}{2 h} g_{\bar{i}}^{*}\left(\mathbf{x}_{b}, t\right)+\frac{1}{2 h} \varepsilon_{B} \varphi_{B} & 1 / 2 \leq h<1\end{cases}
$$

where the definition of quantity $h$ is identical in Eq. (16).

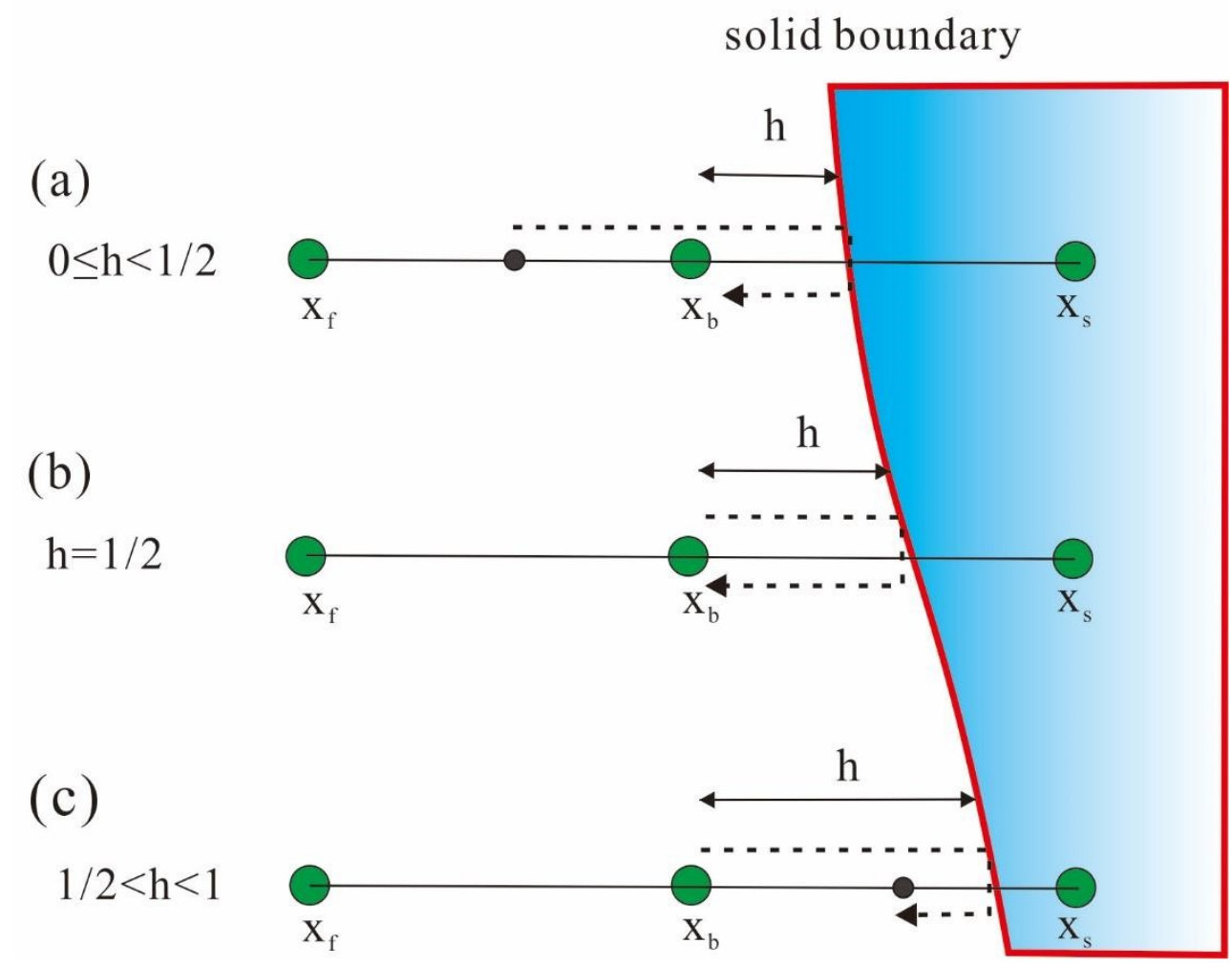

Fig. 4. The detailed cases of interpolated bounce back model. (a) The boundary distance is smaller than half a lattice length. (b) The solid boundary is at the midpoint of a lattice length. (c) The 
boundary distance is larger than half a lattice length.

\subsection{Validation test}

In order to validate the present LBM for the fluid-solid conjugate heat transfer, the effective thermal conductivity obtained from LBM simulation and analytical parallel solution are estimated in a simple three-dimensional cylindrical tube model. As shown in Fig. 5, the geometry of pore phase (cylindrical tube) and solid phase (skeleton) are parallel connection. The simulation domain is $50^{3}$ voxels and its porosity $\phi$ equals to 0.1508 . At the $\mathrm{Z}$ direction, the inlet and outlet of flow field are considered as the pressure boundary and the Dirichlet boundary conditions are set to be $T_{H}=30$ and $T_{L}=10$. The other boundaries are assumed to be adiabatic at the $\mathrm{X}$ and $\mathrm{Y}$ direction. The effective thermal conductivity of the given circular straight capillary tube fitted in a square solid domain (see Fig. 5), could be expressed analytically as [47]

$$
k_{e}=\phi k_{f}+(1-\phi) k_{s}
$$

Meanwhile, the effective thermal conductivity could be estimated from the distribution of temperature flied, which is simulated by LBM simulation [28]:

$$
k_{e}=\frac{\sum q \delta x^{2}}{A\left(T_{H}-T_{L}\right) / L}
$$

where $L$ is the length of cylindrical tube, $A$ is the cross-sectional area, and $q$ is the heat flux of every lattice. Based on the classical Fourier's law, the heat flux can be written as [13]

$$
q=-k \Delta T
$$

where $k$ is the thermal conductivity of solid or fluid phase and $\Delta T$ is the temperature difference of every lattice along the heat transfer direction.

The comparison between the LBM simulation results and the analytical solution results of effective thermal conductivity for different thermal conductivity ratio of the 
solid phase to pore phase $\left(k_{s} / k_{f}\right)$ with porosity $\phi=0.1508$ are shown in Table 2 . As shown in this table, the effective thermal conductivity results of LBM simulation agree excellently with the analytical results, which validates our direct LBM simulation of heat transfer in porous media.

Table 2. The comparison between the effective thermal conductivity from LBM simulation and the analytical solution.

\begin{tabular}{lll}
\hline $\begin{array}{l}\text { Thermal conductivity ratio } \\
\left(k_{s} / k_{f}\right)\end{array}$ & $\begin{array}{ll}\text { LBM simulation results } \\
\left(k_{e} / k_{f}\right)\end{array}$ & $\begin{array}{l}\text { Analytical solution results } \\
\left(k_{e} / k_{f}\right)\end{array}$ \\
\hline 1 & 1.00 & 1.00 \\
2 & 1.85 & 1.85 \\
5 & 4.40 & 4.40 \\
10 & 8.64 & 8.64 \\
20 & 17.13 & 17.13 \\
50 & 42.61 & 42.61 \\
100 & 85.07 & 85.07 \\
200 & 169.99 & 169.99 \\
500 & 424.75 & 424.75 \\
\hline
\end{tabular}




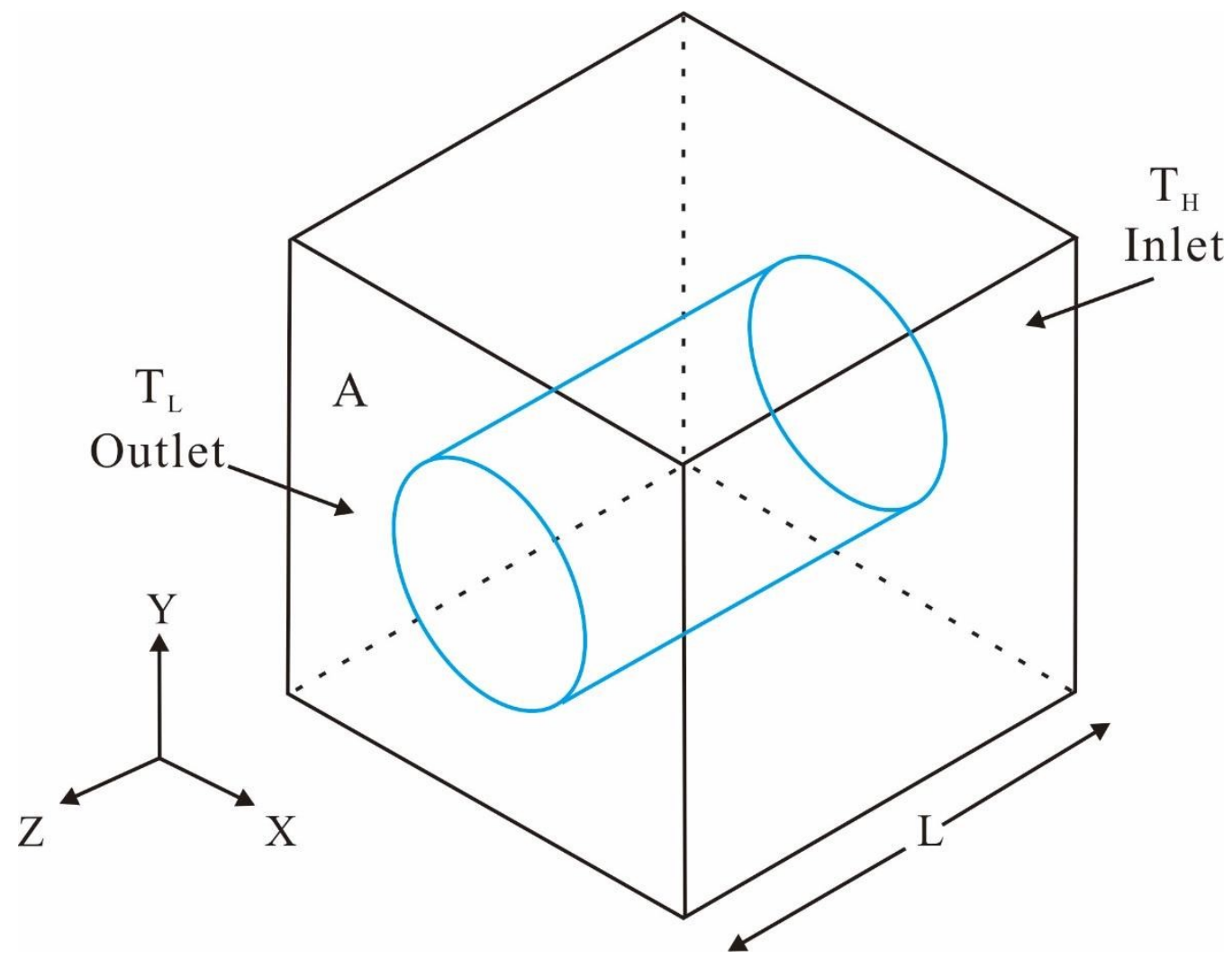

Fig. 5. The geometry and boundary conditions of cylindrical tube model.

\section{Simulation results and discussion}

In this section, the heat transport process in the constructing three-dimensional from Berea sandstone is investigated based on the LBM and fractal model. Moreover, the treatment of boundary conditions for these constructing cubes are identical with the validation model as shown in Fig. 5. The fractal dimensions from these constructed rock images are also determined by box counting method. Furthermore, the influences of a series of essential parameters (such as sample size, fractal dimension, porosity, and thermal conductivity ratio) on heat transfer performance will be analyzed and discussed.

5.1. The influence of computation size on effective thermal conductivity

Physical size or scale effect of transport behaviors is a common phenomenon in porous media. Many transport properties may be dependent on the material size or dimension [48]. The well-known example proposed by Griffith [49] shows that the 
material strength is sensitive to the size of the porous media. In order to study the influence of simulation size on heat transfer in porous media, we constructed different size of three-dimensional rock images, voxel size changing from $50^{3}$ to $300^{3}$, from the Berea sandstone (see Fig. 1); and then we performed detailed LBM simulation of heat transfer process and fractal analysis to obtain their effective thermal conductivity.

Fig. 6 shows the geometries and corresponding steady temperature distribution in the constructed three-dimensional rock images when the thermal conductivity ratio of the solid phase to fluid phase equals to $5\left(k_{s} / k_{f}=5\right)$. It is observed that the temperature distribution contours are much more tortuous and changeable at the interfaces of fluid phase and solid phase than other positions. This is because that the different thermal conductivity results in different heat transfer rate of the fluid phase and solid phase, which may generate the temperature turning points at the interfaces of fluid phase and solid phase. These agree with the expected results given by Cai and Huai [25].

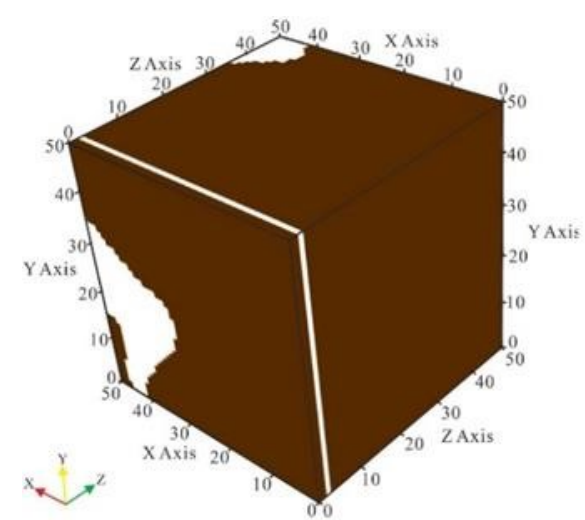

(a)

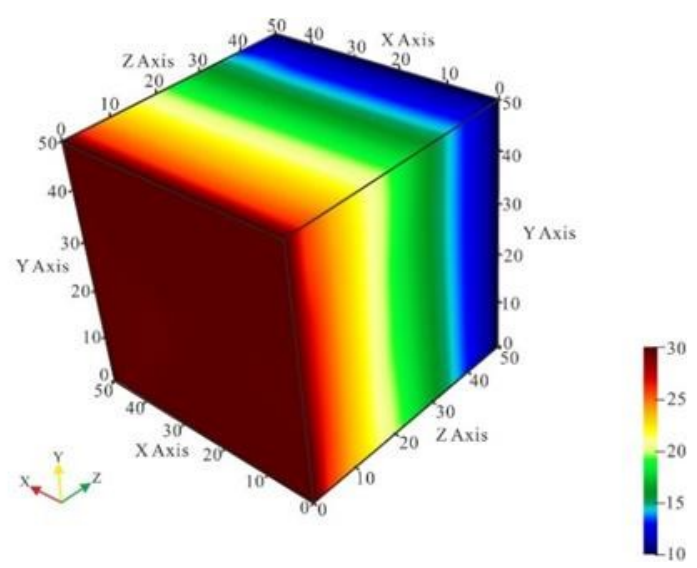



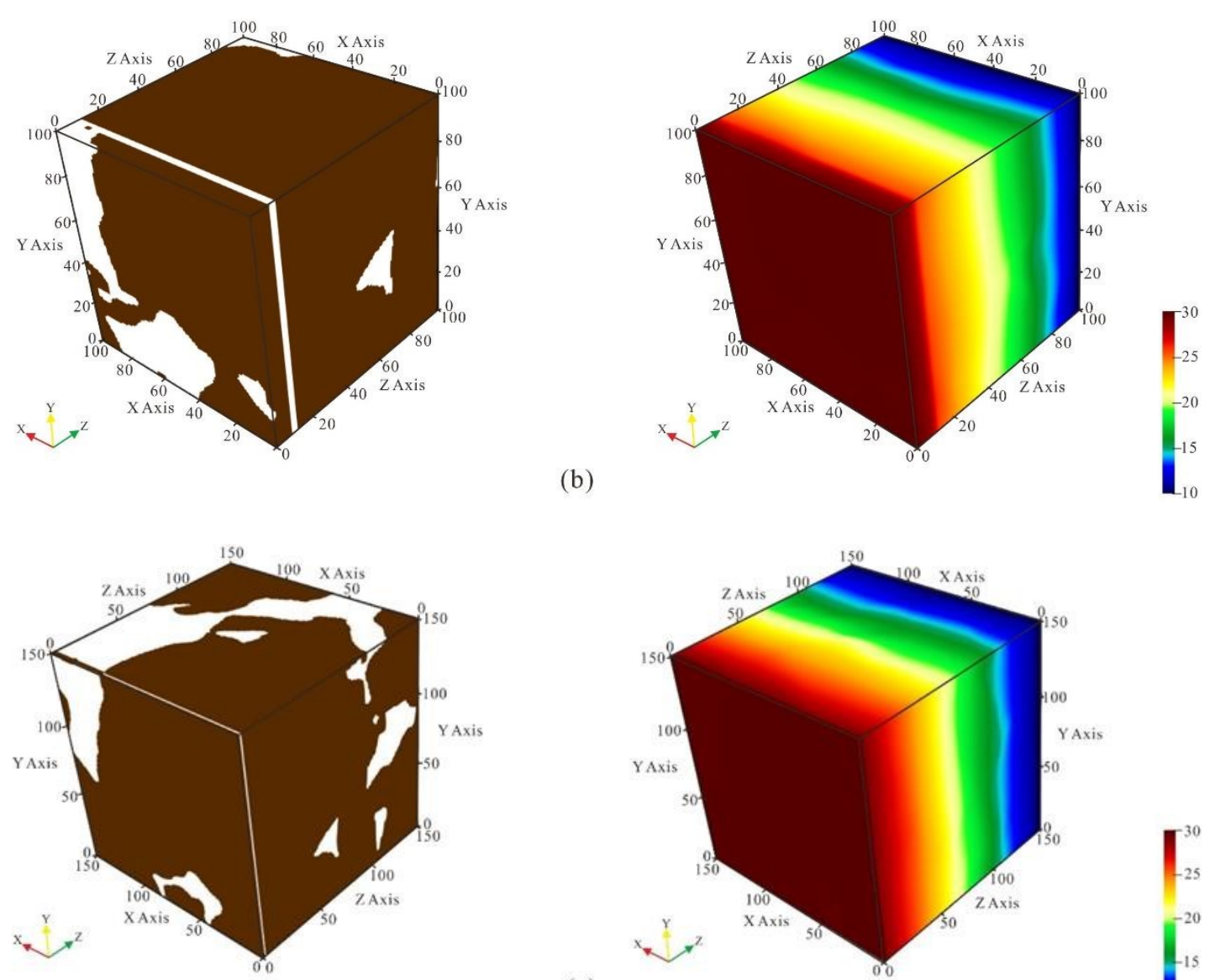

(b)
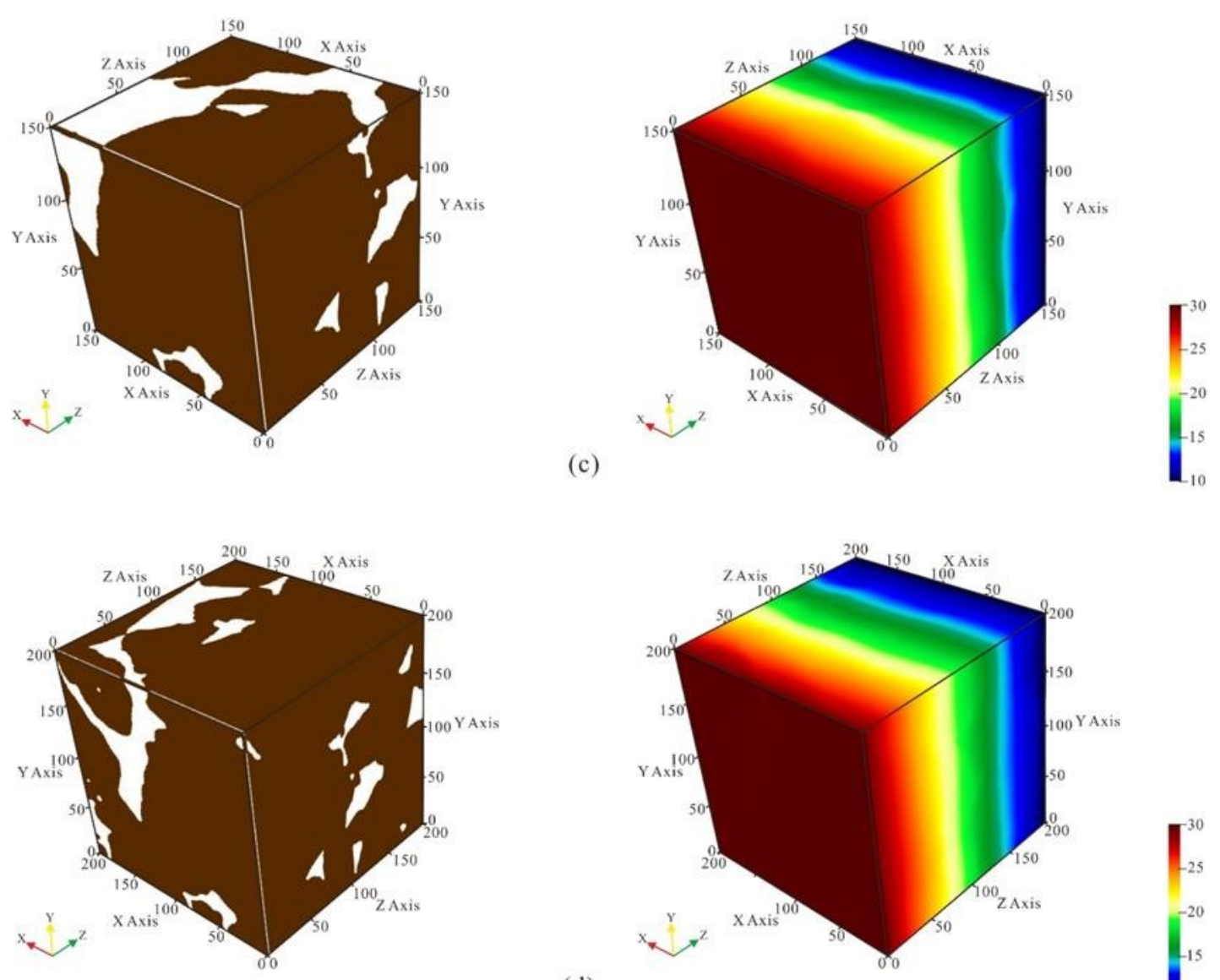

(c)

(d)
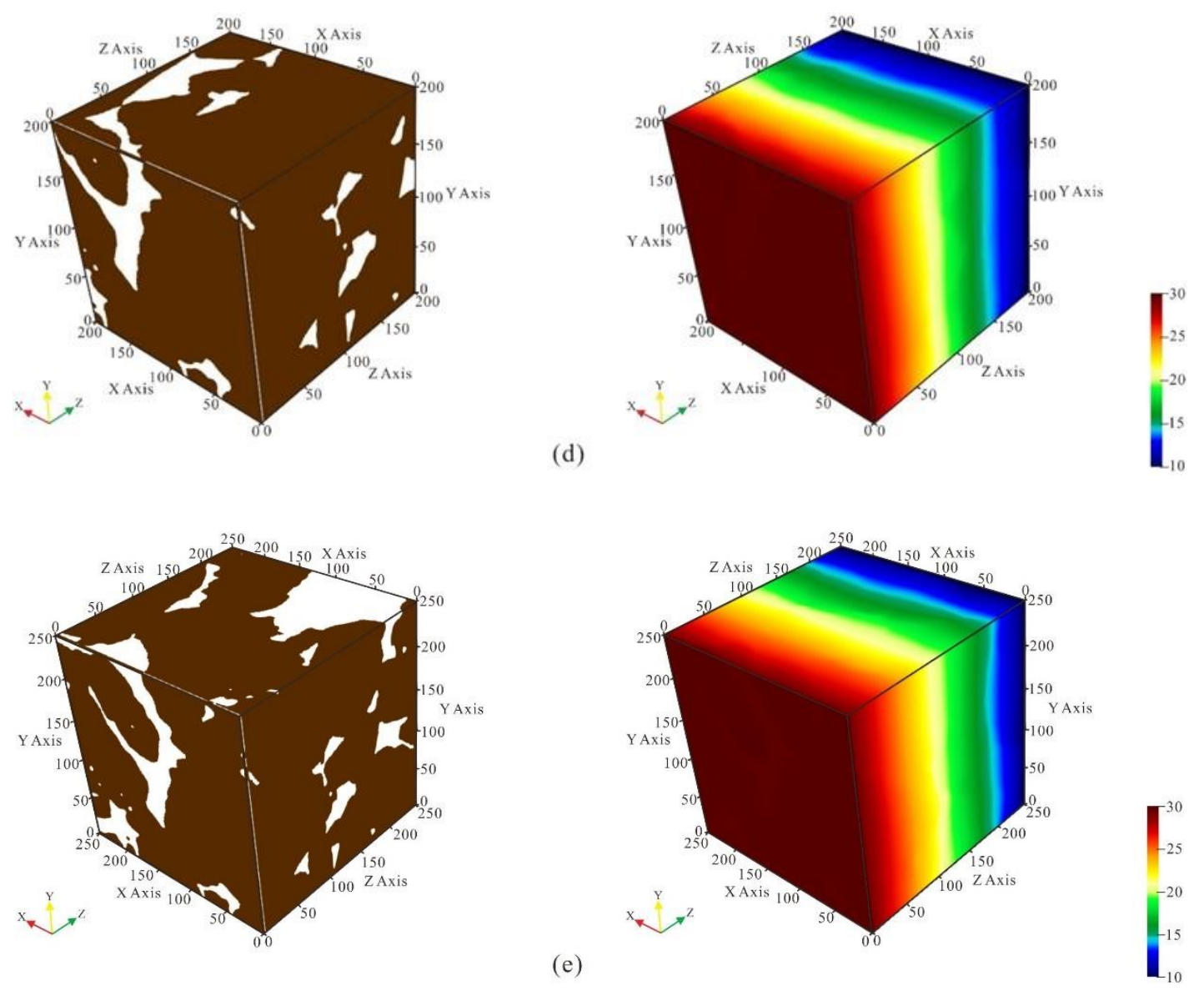


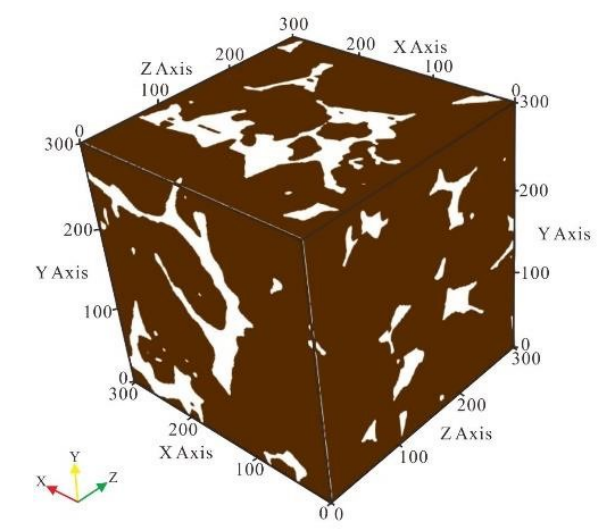

(f)

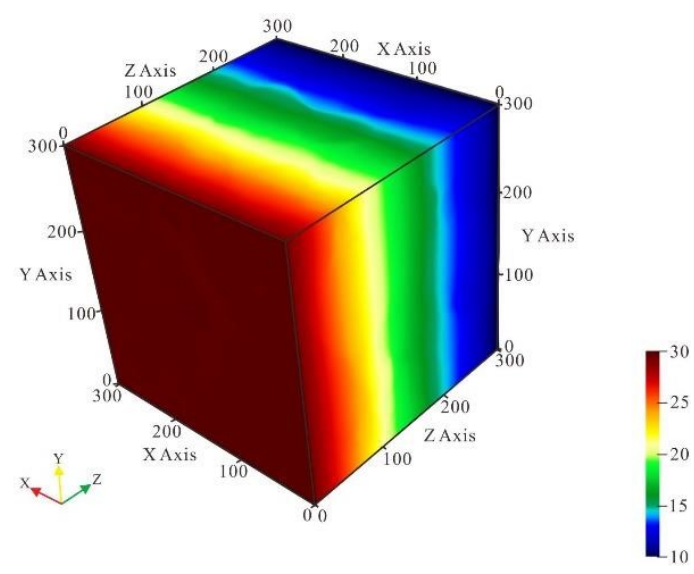

Fig. 6. The geometries and corresponding steady temperature distribution contours of threedimensional constructed sandstone images. Left column: the pore-solid geometry and white and brown are pore phase and solid phase, respectively; right column: the steady temperature distribution. (a) The sample size of $50^{3}$ pixels. (b) The sample size of $100^{3}$ pixels. (c) The sample size of $150^{3}$ pixels. (d) The sample size of $200^{3}$ pixels. (e) The sample size of $250^{3}$ pixels. (f) The sample size of $300^{3}$ pixels.

The LBM simulated and analytical solution (fractal model and Maxwell model) results of effective thermal conductivity as a function of the length size of the rock images with $k_{s} / k_{f}=5$ is presented in Fig. 7.The well-known Maxwell model of effective thermal conductivity is derived from the solution of Laplace's equation and can be written as [47]

$$
k_{e}=k_{f} \frac{3 k_{s}-2\left(k_{s}-k_{f}\right) \phi}{3 k_{f}+\left(k_{s}-k_{f}\right) \phi}
$$

As shown in Fig. 7, the effective thermal conductivity fluctuates at small size and remain constant when the length size of rock image is larger than 250 , which denotes for the representative elementary volume (REV). Comparing with the LBM resolution, the fractal model overestimates the effective thermal conductivity, while the Maxwell model underestimates the effective thermal conductivity. This is because that the Maxwell model is only determined by porosity, but the fractal simulated effective thermal conductivity is very sensitive to the porosity and fractal dimension $D_{s}$. Fig. 8 
shows the fractal dimension $D_{s}$ determined by box counting method versus the variation of cubic sizes. From the Fig. 8, the trend of fractal dimension is similar with the LBM results of dimensionless effective thermal conductivity. It is concluded that the fractal dimension may be a useful parameter to determine the REV of porous media. This result is analogous to the relation between fractal dimension-based REV given by Wu et al. [50].

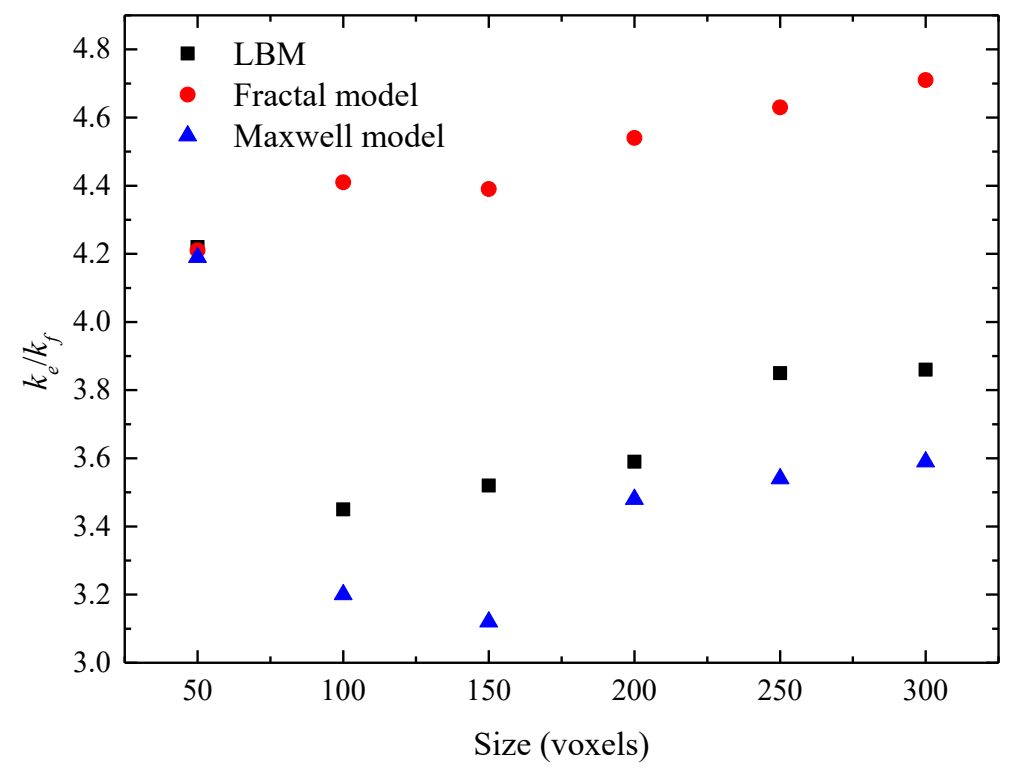

Fig. 7. The influence of rock image size on effective thermal conductivity simulated by LBM and analytical solution (fractal model and Maxwell model) for the constructed three-dimensional rock images with $k_{s} / k_{f}=5$. 


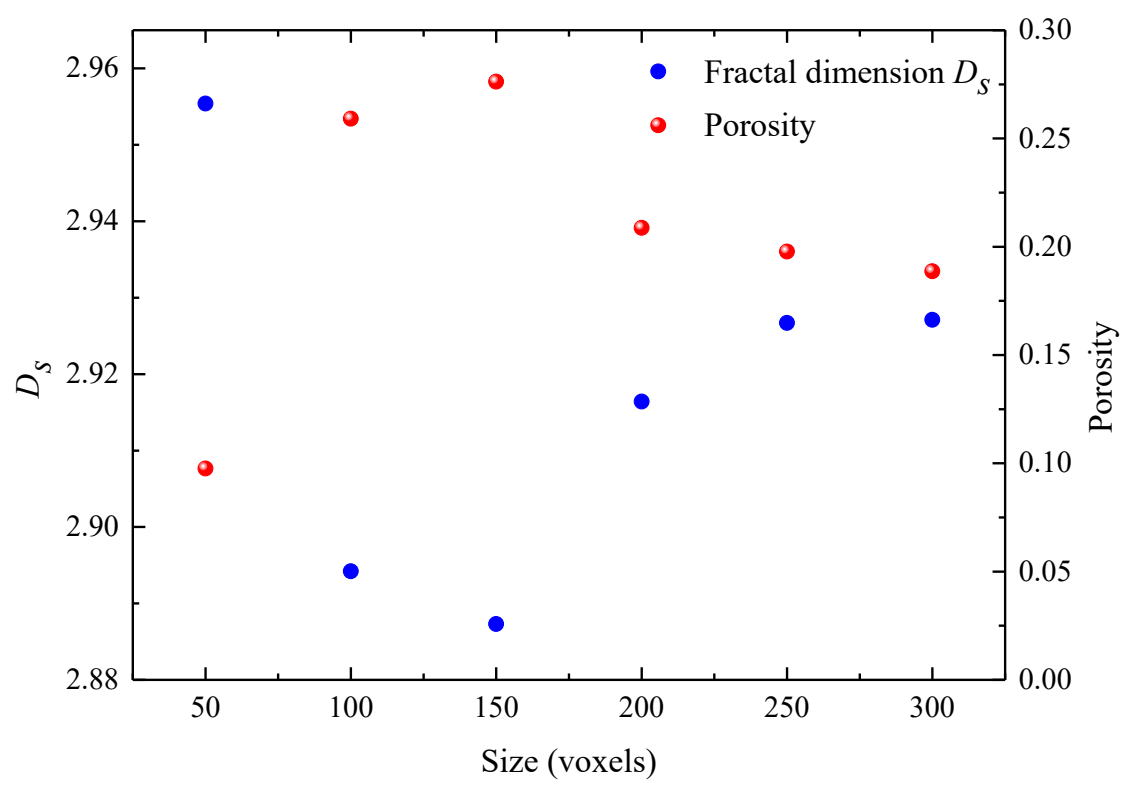

Fig. 8. The fractal dimension determined by box counting method and porosity versus the variation of rock image sizes.

5.2. The influence of porosity on effective thermal conductivity

As shown in fractal based model (Eq. (1)) and Maxwell model (Eq. (23)), porosity is one of the most essential parameters that influence the effective thermal conductivity in saturated porous media. In this subsection, the impact of porosity on effective thermal conductivity is investigate extensively with direct numerical LBM simulation and the results are compared with both Maxwell and fractal model. Subsections with $100^{3}$ voxels are constructed from the Berea sandstone as described in Fig. 1, and the thermal conductivity ratio of the solid phase to fluid phase are set to be $5\left(k_{s} / k_{f}=5\right)$ for all the simulation cases.

The simulated effective thermal conductivity as a function of porosity is presented in Fig. 9. As demonstrated in this figure, the dimensionless effective thermal conductivity $\left(k_{e} / k_{f}\right)$ declines with the increase of porosity for LBM simulations and analytical models. This can be explained by the fact that the fluid phase contribution to effective thermal conductivity increases with the increase of porosity but the fluid phase of thermal conductivity is smaller than solid phase. It has to be noticed that the effective thermal conductivity simulated from LBM simulation and Maxwell model decrease 
much sharper with increasing porosity than that from fractal model. The effective thermal conductivity simulated from LBM and fractal model are more scattered and changeable compared with the Maxwell as the heat transfer also influenced by pore geometries other than porosity in porous media. This situation indicates that the LBM and fractal model can better reflect the heat transfer characteristics with the influence of microstructures in porous media.

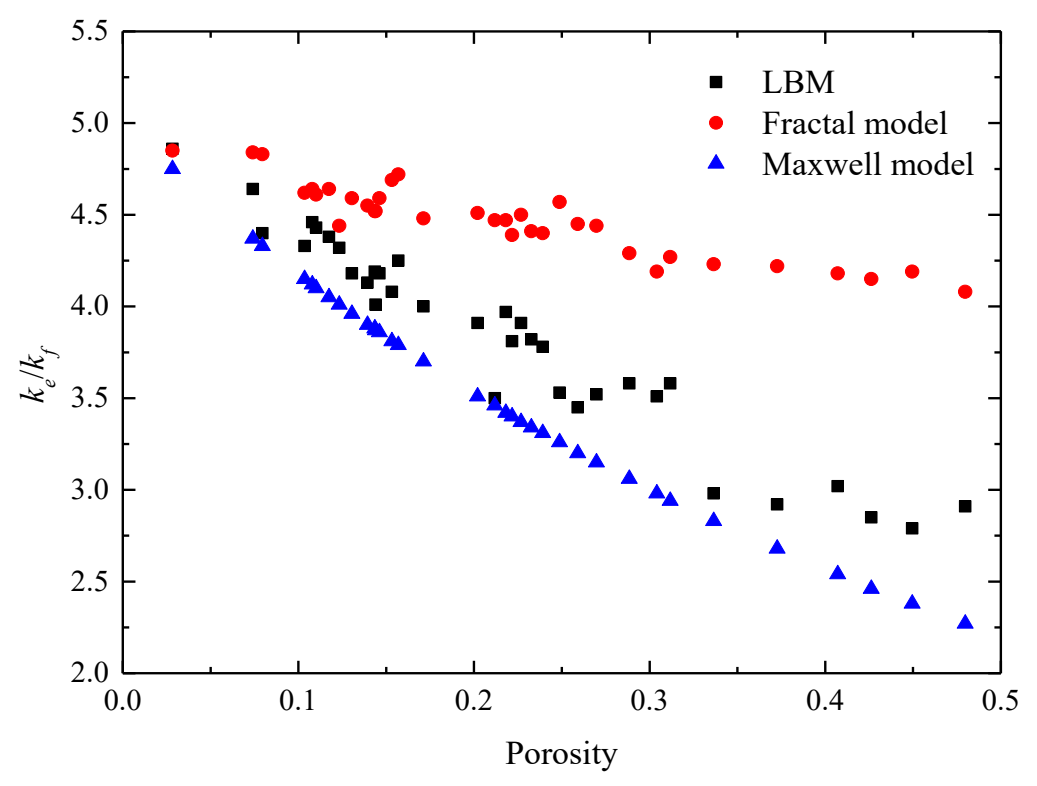

Fig. 9. The influence of porosity on effective thermal conductivity in saturated porous media, here the thermal conductivity ratio of the solid phase to fluid phase equals to $5\left(k_{s} / k_{f}=5\right)$.

Fig. 9 also shows that the difference between the effective thermal conductivity simulated from the three methods increases with the increase of porosity. When the porosity is less than 0.1 , the $k_{e} / k_{f}$ obtained from LBM tends to be equal with the results from fractal model and Maxwell. The reason is that the media may be close proximity to the uniform porous media and the heat transfer process is less effected by the saturated fluid. It can be observed that the $k_{e} / k_{f}$ of fractal model is slightly greater than the results from LBM and Maxwell model as the porosity increases. This discrepancy might be due to the fact that the influences of percolation threshold and contact thermal resistance are not taken into account in fractal model [13]. Recent work 
of effective thermal conductivity model reported by Ghanbarian and Daigle [51] showed that the critical porosity plays an important role to describe the characteristics of heat transfer in porous media. In general, the pore surfaces or contact spots among solid grains are rough in porous media. Recently, Qin et al. [52] proposed an effective thermal conductivity model in fractal porous media with rough surfaces and concluded that the thermal resistance have an effect on the heat transfer in porous media. Another possible source of this discrepancy is that the fractal dimension $D_{s}$ is not accurately calculated by box counting method. Fig. 10 presents the comparison between the fitting results from LBM and box counting method results for fractal dimension. As shown in this figure, it is verified that as expected the fractal dimension $D_{s}$ has an important effect on accurately estimating the effective thermal conductivity in fractal model. The fractal dimension differences between fitting results and box counting method results become slightly larger when the porosity lager than 0.3 . This can be showed that the topology and geometry of pore space and solid phase may become more complicated as the porosity increases, which leads to the more difficulties in determining the fractal dimension accurately. Recently, as suggested by Wu et al. [30], the precise computation of fractal dimension by box counting method may mostly depend on the resolution of micro-CT images and image processing. 


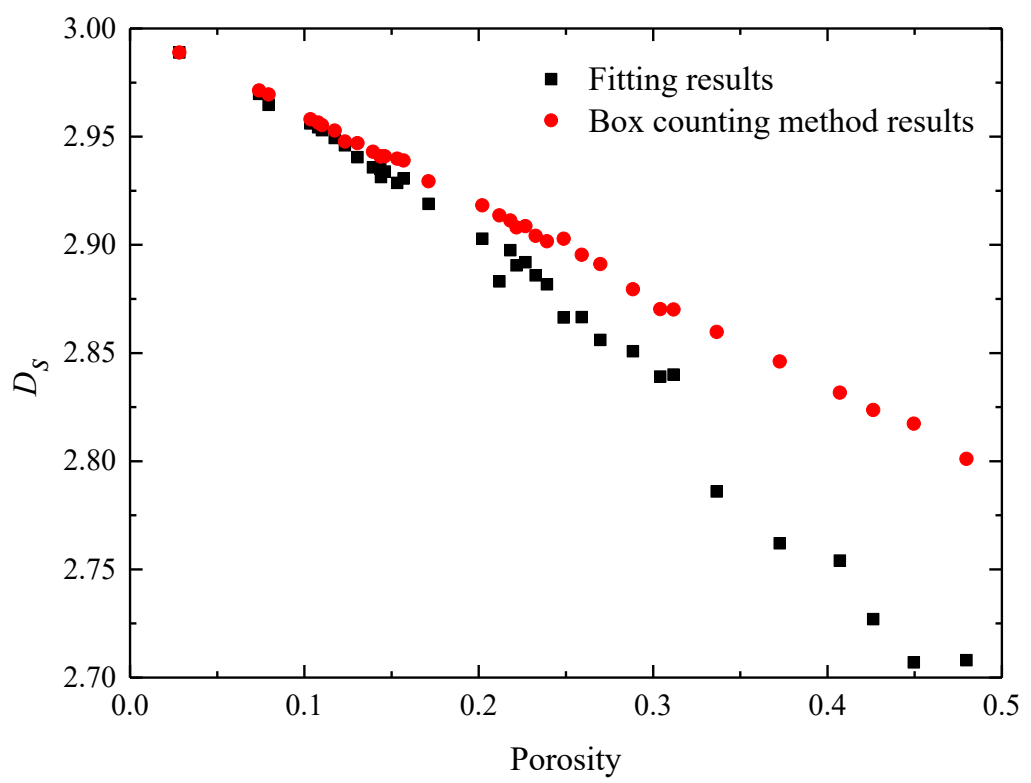

(a)

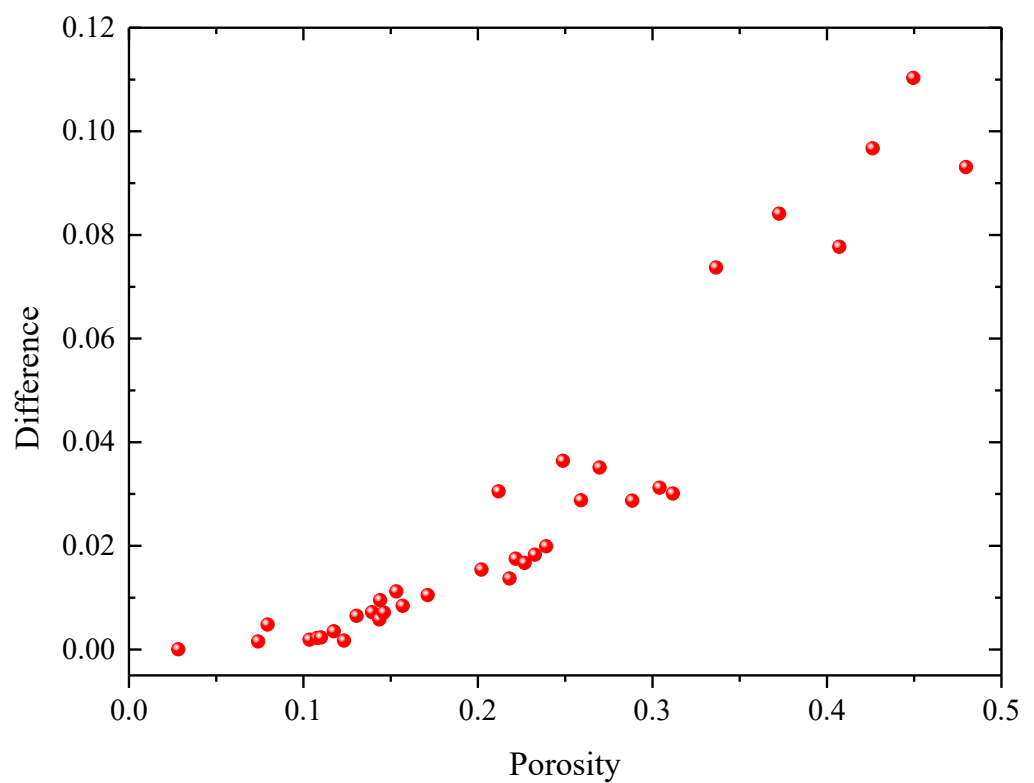

(b)

Fig. 10. The comparison between the fitting results from LBM and box counting method results for fractal dimension $D_{s}$. (a) The fractal dimension from fitting results and box counting method results with different porosities. (b) The fractal dimension difference between fitting results and box counting method results with different porosities.

5.3. The influence of $k_{s} / k_{f}$ on effective thermal conductivity

The effect of various thermal conductivity ratio on effective thermal conductivity is studied in porous media at small, intermediate and large porosities, 0.1035, 0.2487, 
and 0.4071, respectively. Fig. 11 shows the comparison between the LBM simulation results and analytical solution results from fractal model and Maxwell model with different porosities for the different thermal conductivity ratios of the solid phase to fluid phase $\left(k_{s} / k_{f}\right)$. As illustrated in Fig. 10, the results of dimensionless effective thermal conductivity $\left(k_{e} / k_{f}\right)$ decrease with the increase of porosity when $k_{s} / k_{f}>1$. It is also seen that the results of dimensionless effective thermal conductivity from LBM and analytical models have a clearly growing trend with the increase of the thermal conductivity ratio of the solid phase to fluid phase. If $k_{s} / k_{f}>100$, the $k_{e} / k_{f}$ from LBM is larger than the results from analytical models. This is reasonable because the applicability of analytical models for effective thermal conductivity is limited and they may be only suitable for a range of thermal conductivity ratios of the solid phase to fluid phase and porosity or a part of certain geometries of porous media. The present results are consistent with the conclusions given by Wang and Li [53].

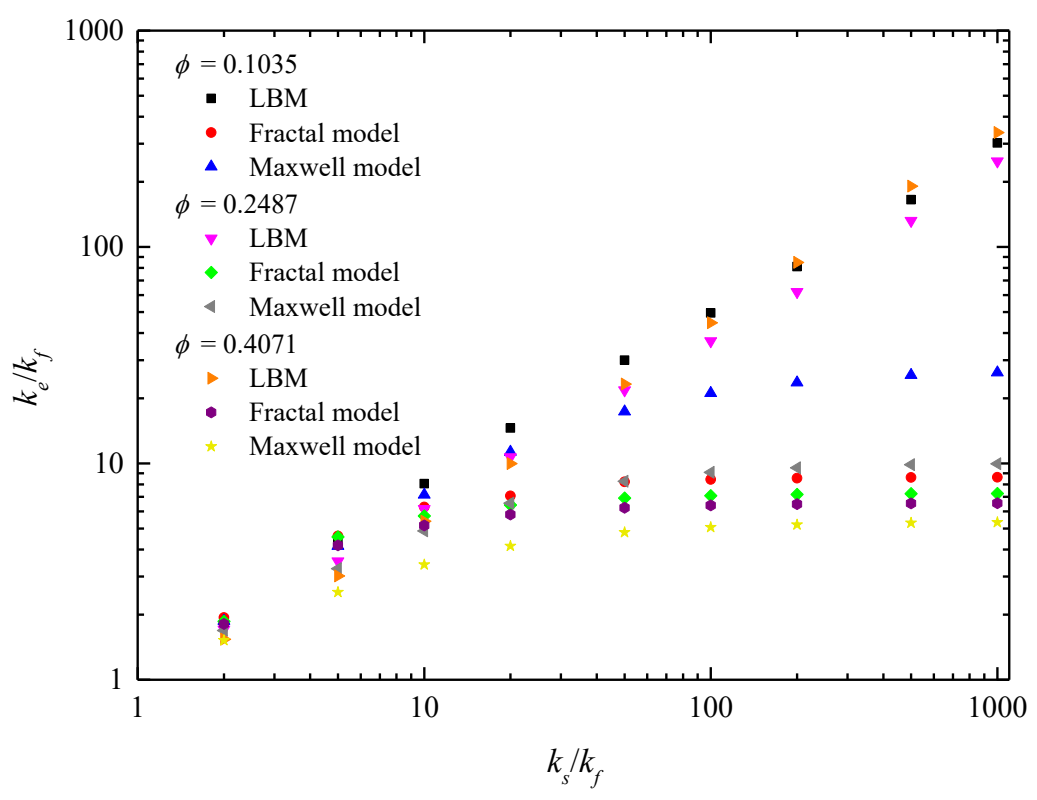

Fig. 11. The influence of thermal conductivity ratios of the solid phase to fluid phase on effective thermal conductivity of saturated porous media.

\section{Summary and conclusions}

In this work, the LBM simulation for fluid-solid coupling and fractal model are 
applied to predict the effective thermal conductivity in three-dimensional saturated porous media. According to the comparison with analytical solution in a simple threedimensional cylindrical tube model, the accuracy of the present LBM simulation is confirmed. The relation between heat transfer property and rock porous media with simulation size, porosity, and thermal conductivity ratios of the solid phase to fluid phase based on the LBM simulation and fractal model is analyzed and discussed extensively. The following essential conclusions can be derived:

(1) The proposed fluid-solid coupling LBM simulation is an efficient tool to describe the heat transfer in complex porous media.

(2) The fractal dimension not only has an important effect on estimating the effective thermal conductivity but also may be a useful parameter to determine the REV of porous media.

(3) Compared with LBM simulation, fractal model slightly over estimate the effective thermal conductivity, while Maxwell model underestimate the effective thermal conductivity. This discrepancy may be due to the influences of percolation threshold and contact thermal resistance are not addressed into the fractal model.

(4) Both LBM simulation and fractal model may reflect a better insight for the physical mechanisms of thermal transport in porous media than conventional models.

It should be noted that the proposed heat transfer model in this study may be applied into other type of rocks and materials in the future work. More effort also should be made to accomplish the experiment about heat transfer in rock porous media.

\section{Acknowledgements}

J. Cai acknowledges support from the National Natural Science Foundation of China, China (No. 41722403), the Hubei Provincial Natural Science Foundation of China, China (No. 2018CFA051), and the Fundamental Research Funds for the Central Universities (China University of Geosciences, Wuhan), China (No. CUGGC04). X. Qin acknowledges support from the China Scholarship Council and British Council's financial for his stay in University of Aberdeen. Y. Zhou acknowledges support from the Royal Society and Royal Society Edinburgh for his travel in China through the International Exchange Program. 


\section{References}

[1] Y.S. Zhao, Z.J. Feng, Z.C. Feng, D. Yang, W.G. Liang, THM (Thermo-hydromechanical) coupled mathematical model of fractured media and numerical simulation of a 3D enhanced geothermal system at $573 \mathrm{~K}$ and buried depth 6000-7000 M, Energy, 82 (2015) 193-205.

[2] Y. Zhou, J. Helland, D.G. Hatzignatiou, Pore-Scale Modeling of Waterflooding in Mixed-Wet-Rock Images: Effects of Initial Saturation and Wettability, SPE Journal, 19 (2014) 88-100.

[3] D. Jougnot, A. Revil, Thermal conductivity of unsaturated clay-rocks, Hydrology And Earth System Sciences, 14 (2010) 91-98.

[4] S. Dai, J.-H. Cha, E.J. Rosenbaum, W. Zhang, Y. Seol, Thermal conductivity measurements in unsaturated hydrate-bearing sediments, Geophysical Research Letters, 42 (2015) 6295-6305.

[5] Y. Wang, C. Ma, Y. Liu, D. Wang, J. Liu, A model for the effective thermal conductivity of moist porous building materials based on fractal theory, International Journal of Heat and Mass Transfer, 125 (2018) 387-399.

[6] B.B. Mandelbrot, The fractal geometry of nature, WH freeman New York, 1983.

[7] J. Cai, B. Yu, A Discussion of the Effect of Tortuosity on the Capillary Imbibition in Porous Media, Transport in Porous Media, 89 (2011) 251-263.

[8] J. Cai, E. Perfect, C.-L. Cheng, X. Hu, Generalized modeling of spontaneous imbibition based on Hagen-Poiseuille flow in tortuous capillaries with variably shaped apertures, Langmuir, 30 (2014) 5142-5151.

[9] B. Yu, P. Cheng, Fractal Models for the Effective Thermal Conductivity of Bidispersed Porous Media, Journal of Thermophysics and Heat Transfer, 16 (2002) 2229.

[10] T. Miao, S. Cheng, A. Chen, B. Yu, Analysis of axial thermal conductivity of dualporosity fractal porous media with random fractures, International Journal of Heat and Mass Transfer, 102 (2016) 884-890.

[11] G. Pia, L. Casnedi, U. Sanna, Porosity and pore size distribution influence on thermal conductivity of yttria-stabilized zirconia: Experimental findings and model predictions, Ceramics International, 42 (2016) 5802-5809.

[12] H.-Q. Jin, X.-L. Yao, L.-W. Fan, X. Xu, Z.-T. Yu, Experimental determination and fractal modeling of the effective thermal conductivity of autoclaved aerated concrete: Effects of moisture content, International Journal of Heat and Mass Transfer, 92 (2016) 589-602.

[13] X. Qin, J. Cai, P. Xu, S. Dai, Q. Gan, A fractal model of effective thermal conductivity for porous media with various liquid saturation, International Journal of Heat and Mass Transfer, 128 (2019) 1149-1156.

[14] B.M. Yu, J.C. Cai, M.Q. Zou, On the Physical Properties of Apparent Two-Phase Fractal Porous Media, Vadose Zone Journal, 8 (2009) 177-186.

[15] P. Xu, S. Qiu, B. Yu, Z. Jiang, Prediction of relative permeability in unsaturated porous media with a fractal approach, International Journal of Heat and Mass Transfer, 64 (2013) 829-837. 
[16] J. Kou, F. Wu, H. Lu, Y. Xu, F. Song, The effective thermal conductivity of porous media based on statistical self-similarity, Physics Letters A, 374 (2009) 62-65.

[17] B. Xiao, Y. Yang, L. Chen, Developing a novel form of thermal conductivity of nanofluids with Brownian motion effect by means of fractal geometry, Powder Technology, 239 (2013) 409-414.

[18] F.A. Soomro, A. Zaib, H. Rizwan U1, M. Sheikholeslami, Dual nature solution of water functionalized copper nanoparticles along a permeable shrinking cylinder: FDM approach, International Journal of Heat and Mass Transfer, 129 (2019) 1242-1249.

[19] B. Li, W. Xu, F. Tong, Measuring thermal conductivity of soils based on least squares finite element method, International Journal of Heat and Mass Transfer, 115 (2017) 833-841.

[20] M. Sheikholeslami, M. Jafaryar, A. Shafee, Z. Li, R.-u. Haq, Heat transfer of nanoparticles employing innovative turbulator considering entropy generation, International Journal of Heat and Mass Transfer, 136 (2019) 1233-1240.

[21] J. Wang, M. Wang, Z. Li, A lattice Boltzmann algorithm for fluid-solid conjugate heat transfer, International Journal of Thermal Sciences, 46 (2007) 228-234.

[22] J. Yang, J. Crawshaw, E.S. Boek, Quantitative determination of molecular propagator distributions for solute transport in homogeneous and heterogeneous porous media using lattice Boltzmann simulations, Water Resources Research, 49 (2013) 85318538 .

[23] K. Timm, H. Kusumaatmaja, A. Kuzmin, The lattice Boltzmann method: principles and practice, in, Springer: Berlin, Germany, 2016.

[24] C.K. Aidun, J.R. Clausen, Lattice-Boltzmann Method for Complex Flows, Annual Review of Fluid Mechanics, 42 (2010) 439-472.

[25] J. Cai, X. Huai, Study on fluid-solid coupling heat transfer in fractal porous medium by lattice Boltzmann method, Applied Thermal Engineering, 30 (2010) 715 723.

[26] M. Basha, N.A.C. Sidik, M.h. Beriache, Numerical simulation of fluid flow and heat transfer in rotating channels using parallel lattice Boltzmann method, International Journal of Heat and Mass Transfer, 115 (2017) 158-168.

[27] H. Lamarti, M. Mahdaoui, R. Bennacer, A. Chahboun, Numerical simulation of mixed convection heat transfer of fluid in a cavity driven by an oscillating lid using lattice Boltzmann method, International Journal of Heat and Mass Transfer, 137 (2019) 615-629.

[28] W.-Z. Fang, L. Chen, J.-J. Gou, W.-Q. Tao, Predictions of effective thermal conductivities for three-dimensional four-directional braided composites using the lattice Boltzmann method, International Journal of Heat and Mass Transfer, 92 (2016) 120-130.

[29] J.S. Gomez, R.J. Chalaturnyk, G. Zambrano-Narvaez, Experimental Investigation of the Mechanical Behavior and Permeability of 3D Printed Sandstone Analogues Under Triaxial Conditions, Transport in Porous Media, 129 (2019) 541-557.

[30] H. Wu, Y. Zhou, Y. Yao, K. Wu, Imaged based fractal characterization of microfracture structure in coal, Fuel, 239 (2019) 53-62.

[31] A. Roy, E. Perfect, W.M. Dunne, L.D. McKay, Fractal characterization of fracture 
networks: An improved box-counting technique, Journal of Geophysical Research: Solid Earth, 112 (2007).

[32] E. Perfect, B. Donnelly, Bi-phase box counting: an improved method for fractal analysis of binary images, Fractals, 23 (2015) 1540010.

[33] J. Cui, D. Liu, Y. Cai, Z. Pan, Y. Zhou, Insights into fractures and minerals in subbituminous and bituminous coals by FESEM-EDS and X-ray $\mu$-CT, Fuel, 237 (2019) 977-988.

[34] P. Lallemand, L.-S. Luo, Theory of the lattice Boltzmann method: Dispersion, dissipation, isotropy, Galilean invariance, and stability, Physical Review E, 61 (2000) 6546-6562.

[35] P.V. Coveney, S. Succi, D. d'Humières, I. Ginzburg, M. Krafczyk, P. Lallemand, L.-S. Luo, Multiple-relaxation-time lattice Boltzmann models in three dimensions, Philosophical Transactions of the Royal Society of London. Series A: Mathematical, Physical and Engineering Sciences, 360 (2002) 437-451.

[36] B. Kravets, H. Kruggel-Emden, Investigation of local heat transfer in random particle packings by a fully resolved LBM-approach, Powder Technology, 318 (2017) 293-305.

[37] Z. Li, M. Yang, Y. Zhang, Lattice Boltzmann method simulation of 3-D natural convection with double MRT model, International Journal of Heat and Mass Transfer, 94 (2016) 222-238.

[38] R. Mei, W. Shyy, D. Yu, L.-S. Luo, Lattice Boltzmann Method for 3-D Flows with Curved Boundary, Journal of Computational Physics, 161 (2000) 680-699.

[39] H.-W. Chang, P.-Y. Hong, L.-S. Lin, C.-A. Lin, Simulations of flow instability in three dimensional deep cavities with multi relaxation time lattice Boltzmann method on graphic processing units, Computers \& Fluids, 88 (2013) 866-871.

[40] H. Sajjadi, A. Amiri Delouei, M. Sheikholeslami, M. Atashafrooz, S. Succi, Simulation of three dimensional MHD natural convection using double MRT Lattice Boltzmann method, Physica A: Statistical Mechanics and its Applications, 515 (2019) 474-496.

[41] Y. Peng, C. Shu, Y.T. Chew, A 3D incompressible thermal lattice Boltzmann model and its application to simulate natural convection in a cubic cavity, Journal of Computational Physics, 193 (2004) 260-274.

[42] Q. Zou, X. He, On pressure and velocity boundary conditions for the lattice Boltzmann BGK model, Physics of Fluids, 9 (1997) 1591-1598.

[43] D.P. Ziegler, Boundary conditions for lattice Boltzmann simulations, Journal of Statistical Physics, 71 (1993) 1171-1177.

[44] M.h. Bouzidi, M. Firdaouss, P. Lallemand, Momentum transfer of a Boltzmannlattice fluid with boundaries, Physics of Fluids, 13 (2001) 3452-3459.

[45] H. Yoshida, M. Nagaoka, Multiple-relaxation-time lattice Boltzmann model for the convection and anisotropic diffusion equation, Journal of Computational Physics, 229 (2010) 7774-7795.

[46] L. Li, R. Mei, J.F. Klausner, Boundary conditions for thermal lattice Boltzmann equation method, Journal of Computational Physics, 237 (2013) 366-395.

[47] J.K. Carson, S.J. Lovatt, D.J. Tanner, A.C. Cleland, Thermal conductivity bounds 
for isotropic, porous materials, International Journal of Heat and Mass Transfer, 48 (2005) 2150-2158.

[48] M. Wang, N. Pan, Predictions of effective physical properties of complex multiphase materials, Materials Science and Engineering: R: Reports, 63 (2008) 1-30. [49] A.A. Griffith, VI. The phenomena of rupture and flow in solids, Philosophical transactions of the royal society of london. Series A, containing papers of a mathematical or physical character, 221 (1921) 163-198.

[50] H. Wu, Y. Yao, Y. Zhou, F. Qiu, Analyses of representative elementary volume for coal using X-ray $\mu-\mathrm{CT}$ and FIB-SEM and its application in permeability predication model, Fuel, 254 (2019) 115563.

[51] B. Ghanbarian, H. Daigle, Thermal conductivity in porous media: Percolationbased effective-medium approximation, Water Resources Research, 52 (2016) 295-314. [52] X. Qin, Y. Zhou, A.P. Sasmito, An effective thermal conductivity model for fractal porous media with rough surfaces, Advances in Geo-Energy Research, 3 (2019) 149155.

[53] F. Wang, X. Li, The stagnant thermal conductivity of porous media predicted by the random walk theory, International Journal of Heat and Mass Transfer, 107 (2017) 520-533. 
Click here to access/download RDM Data Profile XML

DataProfile_4756461.xml 
Conflict of Interest

\section{Conflict of Interest}

We declare that we have no conflict of interest. 